\title{
Photoinduced magnetism in the ferromagnetic semiconductors
}

\author{
Subodha Mishra and Sashi Satpathy \\ Department of Physics \& Astronomy, University of Missouri, Columbia, MO 65211, USA
}

(Dated: June 23, 2018)

\begin{abstract}
We study the enhancement of the magnetic transition temperature $T_{c}$ due to incident light in ferromagnetic semiconductors such as EuS. The photoexcited carriers mediate an extra ferromagnetic interaction due to the coupling with the localized magnetic moments. The Hamiltonian consists of a Heisenberg model for the localized moments and an interaction term between the photoexcited carriers and the localized moments. The model predicts a small enhancement of the transition temperature in semi-quantitative agreement with the experiments.
\end{abstract}

PACS numbers: PACS: 75.50.Pp

There is a class of materials which are ferromagnetic semiconductors $1,2,3,4,5$ such as the Europium chalcogenides $\operatorname{EuX}(\mathrm{X}=\mathrm{S}, \mathrm{O}$, Se, Te) and chalcogenide spinels $\mathrm{MCr}_{2} \mathrm{Y}_{4}(\mathrm{M}=\mathrm{Hg}, \mathrm{Cd} ; \mathrm{Y}=\mathrm{S}, \mathrm{Se})$. The ferromagnetism in these materials are generally well described by the Heisenberg model. In this paper, we are concerned with the effect of incident light on the magnetism, in particular, on the ferromagnetic transition temperature $T_{c}$. The basic mechanism of the photoenhanced magnetism is that light excites electron-hole pairs, which mediate an extra magnetic interaction between the local magnetic moments, on top of any pre-existing magnetic interaction, thereby enhancing the $T_{c}$. The related phenomena, where ferromagnetism occurs only if light is present but otherwise the system is paramagnetic, has been studied in connection with the dilute magnetic semiconductors,$\frac{9,10,11,12,13,14,15,16}{}$

Here we study the photoinduced magnetism in the ferromagnetic semiconductors by solving a model Hamiltonian within a mean-field approach by constructing a BCS-type wave function for the electron-hole pairs, 17 similar to our analysis developed earlier for the dilute magnetic semiconductors ${ }^{14}$ Our work provides a semi-quantitative explanation for the enhancement of $T_{c}$, which has been observed to be as large as $0.16 K$ for EuS6,7,8 under intense laser radiation.

The ferromagnetic semiconductor is modeled by isotropic conduction and valence bands interacting with fixed Eu magnetic moments originating from the f electrons. The bands are spin-split through an exchange interaction with the magnetic ions as indicated in Fig. 1. The Hamiltonian consists of a Heisenberg interaction term between the fixed Eu magnetic moments and a term describing the itinerant band electrons and their interaction with light. We thus write

$$
\mathcal{H}=-\sum_{<i j>} J_{M} S_{i} . S_{j}+\mathcal{H}_{e h}^{L}
$$

where the first term is the Heisenberg term and $\langle i j\rangle$ denotes summation over distinct pairs of nearest neighbours. Each Eu atom has twelve nearest neighbor in the fcc lattice of EuS. The second term $\mathcal{H}_{e h}^{L}$ describes electrons and holes making up the conduction and valence

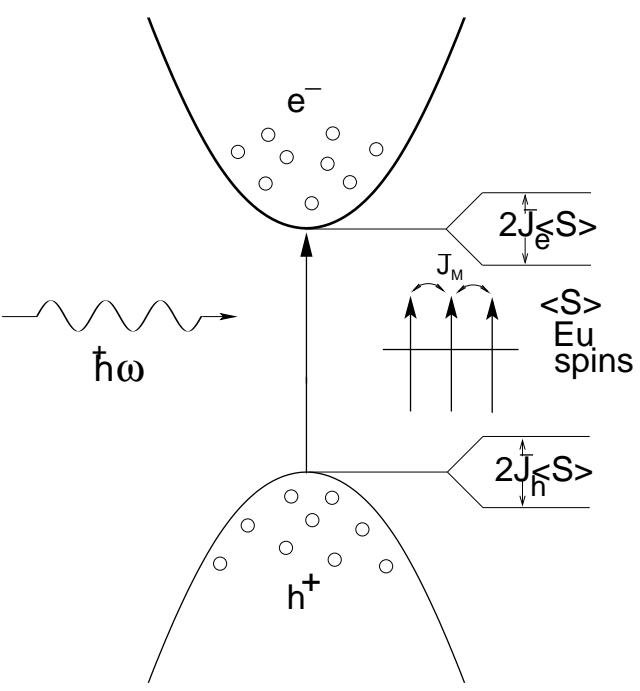

FIG. 1: A schematic picture of the photoinduced ferromagnetism in EuS. Incident light creates electron-hole pairs that mediate an extra ferromagnetic exchange interaction between the Eu moments. The net result is an enhancement of the critical temperature $T_{c}$ by a fraction of a degree $\mathrm{K}$ for typical parameters.

bands and their interaction with light

$$
\mathcal{H}_{e h}^{L}=\mathcal{H}_{k i n}+\mathcal{H}_{c}+\mathcal{H}_{L}(t)
$$

The kinetic energy term is given by

$$
\mathcal{H}_{k i n}=\sum_{k \sigma}\left(E_{k \sigma}^{e} c_{k \sigma}^{\dagger} c_{k \sigma}+E_{k \sigma}^{h} d_{k \sigma}^{\dagger} d_{k \sigma}\right)
$$

where $c_{k \sigma}^{\dagger}, c_{k \sigma}$ and $d_{k \sigma}^{\dagger}, d_{k \sigma}$ are the field operators for the electrons and holes, respectively, with $k$ being the Bloch momentum and $\sigma$, the spin index. Throughout this paper, electrons refer to the photoexcited electrons in the conduction bands and holes refer to the lack of an electron in the valence bands. Since the photon has zero spin and negligible momentum, absorption of light involves a vertical transition of an electron in the valence bands into the conduction bands with the spin of the electron conserved thus resulting in the creation of an electron-hole 
pair described by $c_{k \sigma}^{\dagger} d_{-k-\sigma}^{\dagger}$. Without incident light, we have the "vacuum state" with no electrons nor holes.

The single-particle energies in Eq. 3 include the Zeeman splitting due to the electron interaction with the localized Eu spins, so that

$$
E_{k \sigma}^{e}=E_{g}+\frac{\hbar^{2} k^{2}}{2 m_{e}} \pm J_{e}<S>
$$

and

$$
E_{k \sigma}^{h}=\frac{\hbar^{2} k^{2}}{2 m_{h}} \pm J_{h}<S>
$$

where \pm describes the Zeeman splitting of the up-spin and the down-spin states. Here $J_{e}$ and $J_{h}$ are the electron and hole exchange interactions with the localized $\mathrm{Eu}$ moments, $\langle M\rangle=g \mu_{B}\langle S\rangle$ is the average moment of a Eu atom, $m_{e}$ and $m_{h}$ are the electron and hole masses, respectively, and $E_{g}$ is the band gap.

The Coulomb interaction part consists of three terms

$$
\mathcal{H}_{c}=\mathcal{H}_{e e}+\mathcal{H}_{h h}+\mathcal{H}_{e h}
$$

where the electron-electron interaction term is

$$
\mathcal{H}_{e e}=\frac{1}{2} \sum_{k k^{\prime} q \sigma \sigma^{\prime}} V_{q} c_{k+q, \sigma}^{\dagger} c_{k^{\prime}-q, \sigma^{\prime}}^{\dagger} c_{k^{\prime} \sigma^{\prime}} c_{k \sigma}
$$

with a similar term for the hole-hole interaction, while the electron-hole interaction is given by the expression

$$
\mathcal{H}_{e h}=-\sum_{k k^{\prime} q \sigma \sigma^{\prime}} V_{q} c_{k+q, \sigma}^{\dagger} d_{k^{\prime}-q, \sigma^{\prime}}^{\dagger} d_{k^{\prime} \sigma^{\prime}} c_{k \sigma}
$$

Finally, the last term in Eq. (2) which describes the coupling to the external electromagnetic field of frequency $\omega$, in the "rotating wave approximation" is given as

$$
\mathcal{H}_{L}(t)=\lambda \sum_{k} \rho_{k}\left[\left(c_{k \uparrow}^{\dagger} d_{-k \downarrow}^{\dagger}+c_{k \downarrow}^{\dagger} d_{-k \uparrow}^{\dagger}\right) e^{-i \omega t}+\text { h.c. }\right] .
$$

As mentioned earlier, the light induces a vertical transition with no net spin change and $\lambda \rho_{k}$ is the coupling strength for the creation of such an electron-hole pair. The coupling strength is given as $\lambda \rho_{k}=e E<$ $\psi_{v k}|\hat{e} \cdot \vec{p}| \psi_{c k}>/ m \omega$ where $\psi_{c k}, \psi_{v k}$ are conduction and the valence state wave functions, $\mathrm{E}$ is the strength of the electric field and $\hat{e}$ is the electric polarization vector and $\vec{p}$ is the momentum operator. The matrix element may be computed once the wave functions are known, but here, for simplicity, we neglect the momentum dependence of the matrix elements, so that $\rho_{k}=1$ and the parameter $\lambda$ indicates the strength of the interaction. For strong laser radiation available in the laboratory, a broadly estimated value used by earlier authors $\frac{16,18}{18} \lambda \approx 0.1 \mathrm{eV}$, which we use here in our numerical calculations.

The time-dependence of Eq. (9) can be eliminated 14,18 by an appropriate unitary transformation $e^{-i S} \mathcal{H} e^{i S}$ where $S=-\frac{\omega t}{2} \sum_{k \sigma}\left(c_{k \sigma}^{\dagger} c_{k \sigma}+d_{k \sigma}^{\dagger} d_{k \sigma}\right)$ and the Hamiltonian becomes

$$
\begin{aligned}
\tilde{\mathcal{H}}= & -\sum_{<i, j>} J_{M} S_{i} \cdot S_{j} \\
& +\sum_{k \sigma}\left(E_{k \sigma}^{e}-\hbar \omega / 2\right) c_{k \sigma}^{\dagger} c_{k \sigma}+\left(E_{k \sigma}^{h}-\hbar \omega / 2\right) d_{k \sigma}^{\dagger} d_{k \sigma} \\
& +\mathcal{H}_{c}+\lambda \sum_{k}\left\{\rho_{k}\left(c_{k \uparrow}^{\dagger} d_{-k \downarrow}^{\dagger}+c_{k \downarrow}^{\dagger} d_{-k \uparrow}^{\dagger}\right)+\text { h.c. }\right\} . \quad(10)
\end{aligned}
$$

Thus the time has been eliminated and the transformed Hamiltonian may be interpreted as a "quasi free energy" with the chemical potential for the creation of an electron-hole pair given by $\mu=\hbar \omega$. One can thus write the transformed Hamiltonian in the form $\tilde{\mathcal{H}}=\mathcal{H}_{Q E}-\mu N$, where $N=\sum_{k \sigma} c_{k \sigma}^{\dagger} c_{k \sigma}$ is the number of electron-hole pairs.

The Coulomb interaction term appearing in the Hamiltonian (7) and (8) is given by $V_{q}=4 \pi e^{2} /\left(\epsilon q^{2}\right)$, but in our discussions below, we replace it, for the sake of simplicity, by a contact interaction term $V(\vec{r})=V_{0} \delta(\vec{r})$ without changing the qualitative physics. Its typical magnitude in semiconductors is $V_{0} \approx 20 \mathrm{eV} . \AA^{3} .14$ Typical value for the exchange parameter is: $J_{M}=2.19 \times 10^{-5} \mathrm{eV}$, which is calculated using the expression for the critical temperature for the Heisenberg Hamiltonian $k_{B} T_{c}=$ $(\nu / 3) J_{M} S(S+1)$. For EuS, $T_{c}=16 K, S=7 / 2$, and the number of nearest neighbours $\nu=12$. The magnitude of $J=J_{e}-J_{h}=-0.036 \mathrm{eV}$ as obtained from estimating $J_{e}$ and $J_{h}$ from the splitting of the spin-up and the spin-down bands in the band structure of EuS. 1

We compute the free energy of the system by using a variational wave function similar to the Bardeen-CooperSchrieffer (BCS) wave function with zero net momentum and spin. The variational wave function consists of a superposition of the electron-hole pairs ${ }^{19,20}$ generated by the coupling to light

$$
\left|\Psi>=\prod_{k \sigma}\left(u_{k \sigma}+v_{k \sigma} c_{k \sigma}^{\dagger} d_{-k-\sigma}^{\dagger}\right)\right| 0>,
$$

where $\mid 0>$ is the intrinsic vacuum state (filled valence and empty conduction bands), $v_{k \sigma}$ is the probability amplitude for the creation of an electron-hole pair with the electron momentum and spin of $k \sigma$ and a hole with the opposite momentum and spin, while $u_{k \sigma}$ is the probability amplitude for the absence of such pair excitation. The normalization condition is, as usual, $u_{k \sigma}^{2}+v_{k \sigma}^{2}=1$, and the coefficients $u_{k \sigma}$ and $v_{k \sigma}$ may be chosen as real. In the present case the localized moments break the symmetry between the spin up and down electrons leading to a net spin polarization of the electron and holes. This in turn affects the net magnetic moment, which then affects the energies of the particles through the Zeeman term, so that all quantities have to be determined in a self-consistent manner.

We now calculate the expectation value of the "free energy" from Eq. (10), which may be rewritten in terms 
of the gap parameter to yield ${ }^{14}$

$$
\begin{aligned}
F_{0} & =\sum_{k \sigma}\left[\left(\xi_{k \sigma}+\Omega_{\sigma} / 2\right) v_{k \sigma}^{2}-\left(\Delta_{\sigma} u_{k \sigma} v_{k \sigma} / 2+\lambda u_{k \sigma} v_{k \sigma}\right)\right] \\
& +\frac{\nu}{2} J_{M}<S>^{2} \times \mathcal{N}
\end{aligned}
$$

where

$$
\Delta_{\sigma}=2 V_{0} \sum_{k^{\prime}} u_{k^{\prime} \sigma} v_{k^{\prime} \sigma}+2 \lambda
$$

and

$$
\Omega_{\sigma}=-2 V_{0} \sum_{k^{\prime}} v_{k^{\prime} \sigma}^{2}
$$

Here, $\nu$ is the number of nearest neighbour (12 for the fcc lattice) and the factor of $1 / 2$ in the last term of Eq. 12 appears to avoid double counting. $\mathcal{N}$ is the total number of sites in the crystal and $\xi_{k \sigma}$ is the energy of the electronhole pair excitation with a spin-up electron and a spindown hole measured with respect to the photon energy

$$
\begin{aligned}
\xi_{k \sigma} & =E_{k, \sigma}^{e}+E_{k,-\sigma}^{h}-\hbar \omega \\
& =E_{g}+\frac{\hbar^{2} k^{2}}{2 m^{*}} \pm J<S>-\hbar \omega,
\end{aligned}
$$

where $J=J_{e}-J_{h}$ and the effective mass of the electronhole pair $m^{*-1}=m_{e}^{-1}+m_{h}^{-1}$. Minimizing the free energy with respect to $v_{k \sigma}, \partial F_{0} / \partial v_{k \sigma}=0$, and using the normalization conditions $u_{k \sigma}^{2}+v_{k \sigma}^{2}=1$, we get the occupation amplitudes $u_{k \sigma}$ and $v_{k \sigma}$, which read

$$
u_{k \sigma}^{2}=\frac{1}{2}\left[1+\frac{\xi_{k \sigma}+\Omega_{\sigma}}{\sqrt{\Delta_{\sigma}^{2}+\left(\xi_{k \sigma}+\Omega_{\sigma}\right)^{2}}}\right]
$$

and

$$
v_{k \sigma}^{2}=\frac{1}{2}\left[1-\frac{\xi_{k \sigma}+\Omega_{\sigma}}{\sqrt{\Delta_{\sigma}^{2}+\left(\xi_{k \sigma}+\Omega_{\sigma}\right)^{2}}}\right] .
$$

We solve the equations (16) and (17) numerically which yield the results for $u_{k \sigma}$ and $v_{k \sigma}$, from which the free energy $F_{0}$ is computed using Eq. (12). The typical pairoccupation amplitudes $u_{k}$ and $v_{k}$ have been shown in Fig. (2). We note that in the limit of small interaction $(V=0$ and $\lambda=0$ ), the free energy (Eq. (12)) boils down to the energy of the noninteracting system of electron-hole pair states occupied up to the chemical potential (i.e., $v_{k \uparrow}^{2}$ and $\bar{v}_{k} \downarrow^{2}$ equal to one below $\hbar \omega$ and zero above).

Once the free energy is computed, the magnetization of the $\mathrm{Eu}$ moments is calculated within the mean-field approximation. Each Eu moment experiences an effective magnetic field $\mathrm{H}_{\text {eff }}$ given by

$$
\mathrm{H}_{e f f}=-\frac{\partial F_{0}(M) / \mathcal{N}}{\partial M},
$$

so that the magnetization is given by the expression

$$
M(T)=g \mu_{B} S B_{J}\left(\beta g \mu_{B} S H_{e f f}(M)\right),
$$

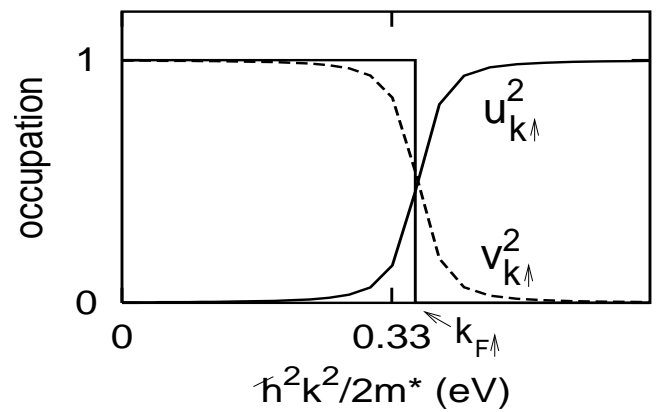

FIG. 2: Electron-hole pair amplitudes $u_{k \uparrow}$ and $v_{k \uparrow}$ as a function of momentum k. Pairs of spin up electrons and spin down holes are filled up to the Fermi momentum $k_{F \uparrow}$ without any interactions. The step function is in the limit of zero interactions $\left(V_{0}=0, \lambda \rightarrow 0\right)$, while both the Coulomb interaction and the coupling to light smear out the Fermi surface. Here parameters are: $\lambda=0.1 \mathrm{eV}, \mathrm{m}^{*}=1.5 \mathrm{~m}_{e}, V_{0}=20$ $\mathrm{eV} . \AA^{3}$ and $\hbar \omega-E_{g}=0.33 \mathrm{eV}, J_{M}=2.19 \times 10^{-5} \mathrm{eV}$ and $J=-0.036 \mathrm{eV}$. The pair amplitudes for the other spin, $u_{k \downarrow}$ and $v_{k \downarrow}$, (not shown in the figure) are slightly shifted to the left, since $k_{F \downarrow}$ is slightly lower than $k_{F \uparrow}$.

where the $\mathrm{B}_{J}$ is the Brillouin function and $\beta=\left(k_{\beta} T\right)^{-1}$ and $M(S)=g \mu_{B} S$. The above two equations are solved self-consistently for a given temperature using the free energy expression Eq. (12).

Note that in our formulation, we have neglected the thermal excitation of the electrons from the valence to the conduction bands, because the number of such carriers is much smaller than the number of the photogenerated carriers. The latter number, obtained by integrating $v_{k \sigma}^{2}$ over all momentum, is typically $\sim 10^{-4} / \AA^{2}$, while the number of intrinsic thermal carriers is many orders of magnitude smaller, ${ }^{23}$ when the semiconductor bandgap is as large as $\sim 1 \mathrm{eV}$, as is the case for EuS.

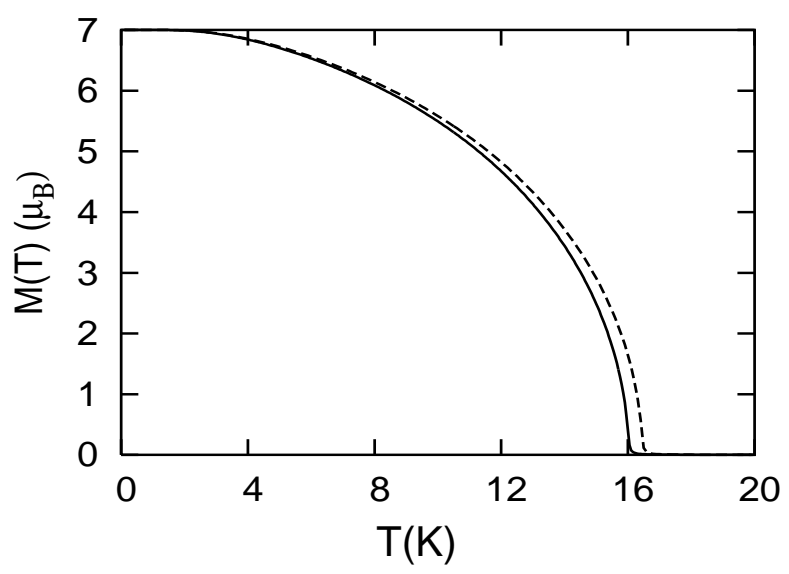

FIG. 3: Dependence of the magnetization $\mathrm{M}$ as a function of temperature $\mathrm{T}$ with (dashed line) and without (solid line) the incident light. Parameters are: $\lambda=0.1 \mathrm{eV}, \mathrm{m}^{*}=1.5$, $V_{0}=20 \mathrm{eV} . \AA^{3}, \hbar \omega-E_{g}=1.5 \mathrm{eV}, J_{M}=2.19 \times 10^{-5} \mathrm{eV}$, and $J=-0.036 \mathrm{eV}$.

The calculated magnetization and the number of pho- 


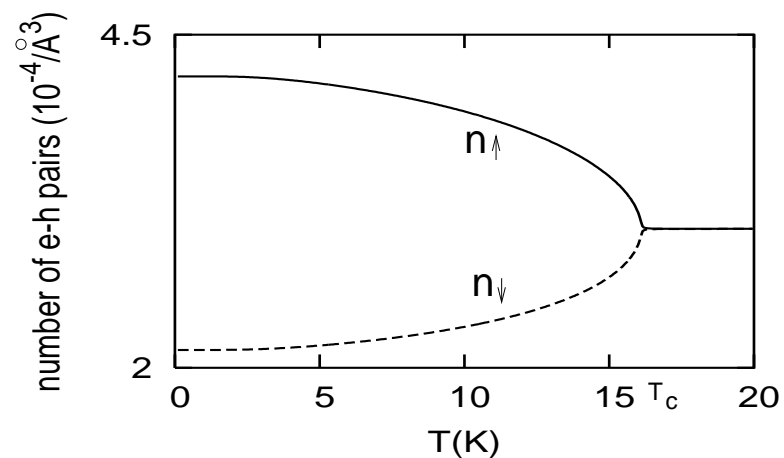

FIG. 4: Pair occupancies as a function of temperature. $n_{\uparrow}$ denotes the number of electron-hole pairs with spin up electrons and spin down holes, while $n_{\downarrow}$ denotes the number of pairs with opposite spins. Below the critical temperature, $n_{\uparrow}$ is larger than $n_{\downarrow}$ because of the lower Zeeman energy of the former pairs. Above $T_{c}$, there is no net megnetization, so that the energy of the electron is independent of the spin (zero Zeeman splitting), so that the pair occupancies are independent of the spin of the electron or hole. Parameters are the same as for Fig. 3

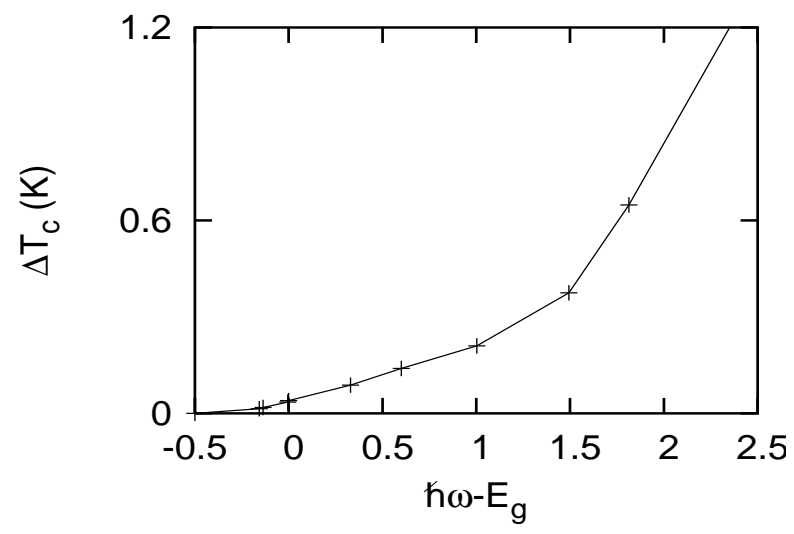

FIG. 5: Photoinduced increase of the magnetic transition temerature as a function of the photon energy. Parameters are the same as in Fig. 2. A larger photon energy leads to a larger number of carriers which add to the ferromagnet

toexcited electron-hole pair as a function of the temperature are shown in the Figs. 3 and 4 respectively. These are the two main results of this paper. As seen from the figures, there is a transition from the paramagnetic to the ferromagnetic state as temperature is decreased. Fig. 3 shows that with the incident light and for typical parameters, the photoinduced increase in the transition temperature $\mathrm{T}_{c}$ is of the order of a fraction of a degree, $\Delta T_{c} \sim 0.2 \mathrm{~K}$, in line with the experimental value ${ }^{8}$
$\Delta T_{c} \sim 0.16 \mathrm{~K}$ for the light frequency of $\hbar \omega-E_{g}=0.33 \mathrm{eV}$.

Fig. 4 shows the number of pairs of spin-up electron and spin-down hole and pairs of spin-down electron and spin-up holes as function of temperature. While the number of the electron-hole pairs is controlled by the chemical potential $(\mu=\hbar \omega)$, the distribution of the pairs between the two different spin types (spin-up electron and spindown hole or vice versa) is determined by the magnitude of $\langle S\rangle$, which affects the pair energy as indicated from Eqs. (15) and (19). The effective magnetic field experienced by the Eu spins is roughly proportional to the difference of the number of the two spin types, as may be seen by keeping the first line in the free energy Eq. (12) and using Eqs. (15) and (18), so that $H_{\text {eff }} \propto\left(n_{\uparrow}-n_{\downarrow}\right)$. This in turn implies that this number difference and the magnetization $\mathrm{M}$ follow one another, both ultimately going to zero at the transition temperature $T_{c}$ as seen from Figs. 3 and 4 In the paramagnetic phase, the photoexcited electron-hole pairs are present, but the two spin types are the same in number.

In Fig. 5 we plot the increase in $T_{c}$ vs. the incident light frequency, which as discussed earlier effectively serves as the chemical potential. We see that as the frequency increases, more and more photocarriers are created and hence $\Delta T_{c}$ increases. The figure shows that even when $\hbar \omega-E_{g}<0$, there is an increase in $T_{c}$. This means that we have photoinduced magnetization even for the sub-bandgap light frequency, an issue that has been studied in detail by previous authors, 16,24 Note that we have neglected the relaxation of the excited carriers, so that in our case carriers are filled up to the chemical potential $\mu=\hbar \omega$ at equilibrium. This relaxation effect will diminish the total number of photoexcited carriers, resulting in a somewhat lower value of $\Delta T_{c}$ than predicted from our analysis. Such relaxation effects must be taken into account if a more quantitative description is desired.

In summary, we studied the photoinduced enhancement of magnetization in the ferromagnetic semiconductors by solving a model Hamiltonian which takes into account the Coulomb interaction, light-matter interaction, the exchange interaction between the carriers and the fixed magnetic moments, as well as the direct exchange interaction among the localized moments. From the calculated free energy, we computed the magnetization of the system as a function of temperature. There is an enhancement of $T_{c}$ by a fraction of a degree $\mathrm{K}$ due to carriers created by light, in semi-quantitative agreement with the observed experimental results for EuS.

This work was supported by the U. S. Department of Energy through Grant No. DE-FG02-00ER45818.
1 W. Muller and W. Nolting, Phys. Rev. B 66085205 (2002).

2 P. Larson and W. L. Lambrecht, J. Phys.:Condensed Matter 1811333 (2006)

${ }^{3}$ W. Lems, P. J. Rijnierse, P. F. Bongers and U. Enz, Phys.
Rev. Lett. bf 211643 (1968)

4 N. Sanford, R. W. Davies, A. Lempicki, W. J. Miniscalco and S. J. Nettel, Phys. Rev. Lett. 501803 (1983)

${ }^{5}$ S. G. Rudov and V. G. Veselago, Sov.Phys.Solid State 21 
1875 (1979)

${ }^{6}$ E. L. Nagaev, Phys. Status Solidi B 145, 11 (1988)

7 M. M. Afanasev, M. E. Kompan and I. A. Merkulov, Zh. eksper. teor. Fiz. 71, 2068 (1976)

8 M. M. Afanasev, M. E. Kompan and I. A. Merkulov, Zh. eksper. teor. Fiz.,Pisma 23, 621 (1976)

${ }^{9}$ H. Krenn, W. Zawadzki and G. Bauer, Phys. Rev. Lett. 55, 1510 (1985)

10 S. Koshihara, A. Oiwa, M. Hirasawa, S. Katsumoto, Y. Iye, C. Urano, H. Takagi and H. Munekate, Phys. Rev. Lett. 78, 4617 (1997)

11 A. Oiwa, Y. Mitsumori, R. Moriya, T. Slupinski and H. Munekata, Phys. Rev. Lett. 88, 137202-1 (2002)

12 Y. Mitsumori, A. Oiwa, T. Slupinski, H. Maruki, Y. Kashimura, F. Minami and H. Munekata, Phys. Rev. B. 69, 033203 (2004)

13 J. Wang, I. Cotoros, K. M. Dani, X. Liu, J. K. Furdyna, and D. S. Chemla, Phys. Rev. Lett. 98, 217401 (2007)

14 S. Mishra, G. S. Tripathi, and S. Satpathy, Phys. Rev. B 77, 125216 (2008)

15 S. Mishra, Photoinduced Ferromagnetism (VDM Verlag Dr. Mueller e. K., Saarbrücken, 2008)
16 J. Fernandez-Rossier, C. Piermarocchi, P. Chen, A. H. MacDonald and L. J. Sham, Phys. Rev. Lett. 93, 127201 (2004)

17 J. Bardeen, L. N. Cooper, and J. R. Schrieffer, Phys. Rev. B. 108,1175 (1957)

18 C. Comte and G. Mahler, Phys. Rev. B 34, 7164 (1986); C. Comte and G. Mahler, Phys. Rev. B 38, 10517 (1988)

19 Th. Harbich and G. Mahler, Phys. Status Solidi B 104, 185 (1981)

20 Th. Harbich and G. Mahler, Phys. Status Solidi B 117, 635 (1983)

21 F. Bassani and G. Pastori Parravicini, Electronic States and Optical Transitions in Solids (Pergamon, New York, 1975)

${ }^{22}$ V. I. Litvinov, K. A. Valuev, and K. D. Tovstyuk, Sov. Phys. JETP 55, 478 (1982)

23 C. Kittel, Introduction to Solid State Physics, Seventh Ed. (Wiley, 1995)

24 D. Fröhlich, A. Nöthe, and K. Reimann, Phys. Rev. Lett. 55, 1335 (1985) 